\title{
Bioprosthetic mitral valve thrombosis in a patient in sinus rhythm after the radiofrequency maze procedure
}

\author{
Nakao Masakazu, MD, Lim See Lim, MBBS, FRCS, FAMS, and Chua Yeow Leng, MBBS, FRCS, FAMS, Singapore
}

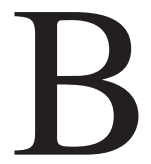

ioprosthetic valves are the preferred option for mitral valve replacement in the elderly because there is a low risk of thromboembolic complications compared with mechanical valves. Current consensus is that long-term anticoagulation can be avoided in patients with bioprosthetic valve replacement because postoperative thromboembolic risk after 3 months is extremely low. ${ }^{1}$ Once anticoagulation is withdrawn, thrombosis causing bioprosthetic valve dysfunction is extremely rare.

\section{Clinical Summary}

A 69-year-old man with a history of stroke 14 years before surgical intervention underwent mitral valve replacement with a $31-\mathrm{mm}$ Hancock II porcine bioprosthesis (Medtronic, Inc, Minneapolis, Minn) and the radiofrequency maze procedure for severe mitral regurgitation and chronic atrial fibrillation. He was started on warfarin, and the international normalized ratio was kept within a therapeutic range of 2.0 to 2.5. Postoperative transthoracic echocardiography (TTE) showed normal bioprosthetic valve function with a mean mitral pressure gradient (PG) of $4.0 \mathrm{~mm} \mathrm{Hg}$.

Warfarin was discontinued at $1 \frac{1}{2}$ years postoperatively because there was no recurrence of atrial fibrillation on follow-up electrocardiography and 24-hour Holter tests. Aspirin was continued for minor coronary artery disease. TTE before cessation of warfarin confirmed normal mitral valve function with a mean PG of 6.0 $\mathrm{mm} \mathrm{Hg}$. Ratio of early filling velocity to atrial contraction velocity (E/A ratio) was 1.58 , implying active atrial contraction. Left ventricular ejection fraction (LVEF) was $62 \%$. Left atrial diameter was $6.0 \mathrm{~cm}$.

One month after warfarin was stopped, the patient was admitted with fever, shortness of breath, and loss of appetite for 3 days. Blood culture was sterile. Chest radiography revealed acute pulmonary edema. TTE demonstrated severe mitral stenosis with a mean mitral PG of $24 \mathrm{~mm} \mathrm{Hg}$ and pressure half-time (PHT) of $187 \mathrm{~ms}$. The mitral valve leaflets were thickened, and leaflet motion was restricted. There was no change in LVEF or left atrial diameter. The E/A ratio was 1.4. Transesophageal echocardiography (TEE) confirmed the findings on TTE. No discrete intracardiac

From the Department of Cardiothoracic Surgery, National Heart Centre, Singapore.

Received for publication July 21, 2006; accepted for publication Aug 8, 2006.

Address for reprints: Nakao Masakazu, National Heart Centre, Singapore, Department of Cardiothoracic Surgery, Mistri Wing, 17 Hospital Ave, Singapore 168752, Singapore (E-mail: mnakao@singnet.com.sg).

J Thorac Cardiovasc Surg 2006;132:1464-5

$0022-5223 / \$ 32.00$

Copyright (C) 2006 by The American Association for Thoracic Surgery doi:10.1016/j.jtcvs.2006.08.018 thrombus was visualized. Valve thickness on TEE was $6.5 \mathrm{~mm}$ (Figures 1 and 2).

Intravenous unfractionated heparin and diuretics were started, and warfarin was resumed. Heparin was withdrawn when the international normalized ratio reached the therapeutic range. Hematologic work-up did not reveal a hypercoagulable state.

A repeat TTE 1 week later showed a decreased mean mitral PG of $11 \mathrm{~mm} \mathrm{Hg}$. PHT had also decreased to $107 \mathrm{~ms}$. Two weeks after treatment was commenced, TTE demonstrated normal mitral bioprosthetic valve function with a mean mitral PG of $6.2 \mathrm{~mm} \mathrm{Hg}$ and a PHT of 114 ms. Three months after discharge, TEE revealed a mean mitral valve PG of $4.0 \mathrm{~mm} \mathrm{Hg}$ and a valve thickness of $1.2 \mathrm{~mm}$.

The patient was maintained on warfarin and was well on follow-up 6 months later.

\section{Discussion}

Bioprosthetic mitral valve thrombosis is a rare cause of prosthetic valve dysfunction postoperatively. Low cardiac output, ${ }^{1}$ large left atrium, ${ }^{1}$ prior history of thromboembolic events, ${ }^{1}$ low transvalvular flow caused by atrial fibrillation, ${ }^{2,3}$ and abnormality in blood rheology ${ }^{4}$ are most commonly cited as predisposing factors.

In this case there were no obvious risk factors for thrombosis: LVEF was normal, the left atrium was only mildly enlarged, and there was no hypercoagulable diathesis, although the patient did have a history of stroke 14 years before the operation. Surgical intervention had also successfully been used to treat the problem of atrial fibrillation, and he had been in sinus rhythm for $1 \frac{1}{2}$ years. His E/A ratio was 1.58 before warfarin was withdrawn. He was also taking aspirin, and it has been reported that aspirin alone can reduce the risk of postoperative thromboembolic complications after

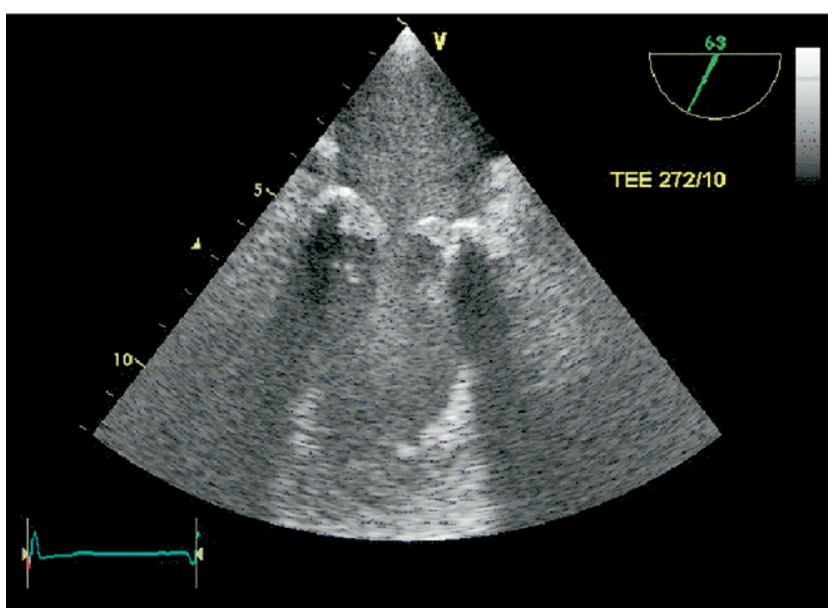

Figure 1. Transesophageal echocardiogram (TEE) before treatment showing grossly thickened mitral valve. 


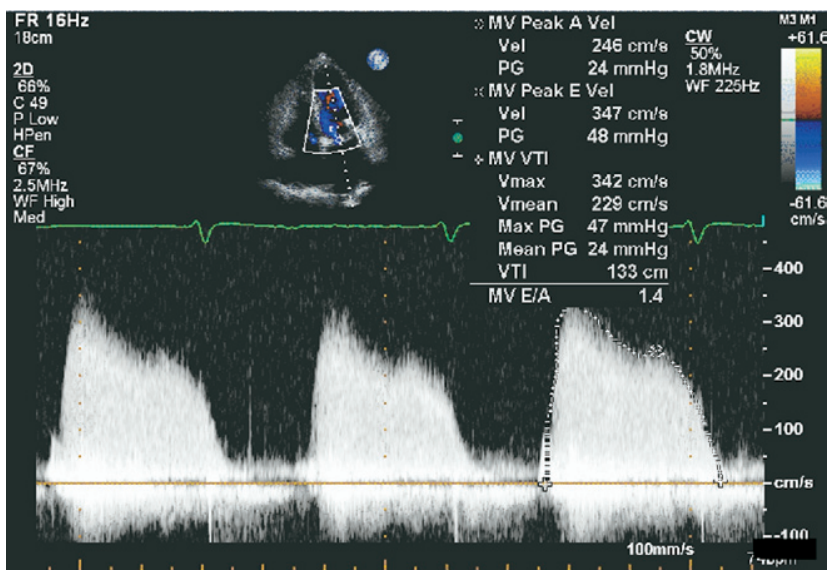

Figure 2. Doppler scan showing increased mean mitral pressure gradient (mean mitral pressure gradient $24 \mathrm{~mm} \mathrm{Hg}$ ).

bioprosthetic valve implantation in the mitral position. ${ }^{1}$ Radiofrequency maze lesion lines were unlikely to be a source leading to propagation onto the valve leaflets because there were no intraatrial clots and thrombus was laminar along all the valve leaflets.

Intravenous heparin was started in this patient with a plan to commence thrombolytic therapy if heparin alone was ineffective. Although thrombolytic therapy is an established first-line treat- ment, this patient responded to heparin anticoagulation followed by warfarin. Several studies have reported good resolution of bioprosthetic thrombus after conventional anticoagulation. ${ }^{2-5}$ Concurring with these studies, we had a favorable result after 1 week of heparin and subsequent warfarinization.

Clinically significant bioprosthetic valve thrombosis is rare, although the reported incidence in the study by Oliver and colleagues ${ }^{5}$ is $6.2 \%$. It should be considered a possible cause of valve dysfunction when there is increasing transvalvular pressure gradient after anticoagulation is terminated. Treatment with conventional anticoagulation is unavoidable in such a setting.

\section{References}

1. Stein PD, Alpert JS, Dalen JE, Horstkotte D, Turpie AG. Antithrombotic therapy in patients with mechanical and biological prosthetic heart valves. Chest. 1998;114(suppl 5):602S-10S.

2. Thomas B, Carreras F, Borras X, Pons-Lladó G. An usual case of bioprosthetic mitral valve thrombosis. Ann Thorac Surg. 2001;72: 259-61.

3. Korkolis DP, Passik CS, Marshalko SJ, Koullias GJ. Early bioprosthetic mitral valve "pseudostenosis" after complete preservation of the native mitral apparatus. Ann Thorac Surg. 2002;74:1689-91.

4. Koppensteiner R, Moritz A, Schlick W, et al. Blood rheology after cardiac valve replacement with mechanical prostheses or bioprostheses. Am J Cardiol. 1991;67:79-83.

5. Oliver JM, Galloge P, Gonzalez A, Dominguez FJ, Gamallo C, Mesa JM. Bioprosthetic mitral valve thrombosis: clinical profile, transesophageal echocardiographic features, and follow-up after anticoagulant therapy. J Am Soc Echocardiogr. 1996;9:691-9.

\section{A case of mitral valve plasty for Libman-Sacks endocarditis mimicking a cardiac tumor}

Shingo Taguchi, MD, Kazuhiro Hashimoto, MD, Yoshimasa Sakamoto, MD, Hiroshi Okuyama, MD, and Shinichi Ishii, MD, Tokyo, Japan

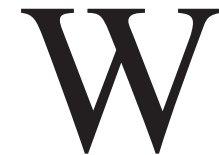
e report a patient with multiple cerebral infarcts in whom 2-dimensional echocardiography showed a large mass attached to the anterior mitral leaflet and chordae. An intracardiac tumor was diagnosed, and surgical intervention was performed. The resected mass

\footnotetext{
From the Department of Cardiovascular Surgery, Jikei University School of Medicine, Tokyo, Japan.

Received for publication May 27, 2006; revisions received June 27, 2006; accepted for publication July 12, 2006.

Address for reprints: Kazuhiro Hashimoto, MD, Department of Cardiovascular Surgery, Jikei University School of Medicine, 3-25-8, Nishishinbashi, Minato-ku, Tokyo, Japan, 105-8461 (E-mail: kaz-hashi@jikei.ac.jp).

J Thorac Cardiovasc Surg 2006; 132:1465-7

$0022-5223 / \$ 32.00$

Copyright $\odot 2006$ by The American Association for Thoracic Surgery doi:10.1016/j.jtcvs.2006.07.034
}

proved to be a Libman-Sacks vegeta-

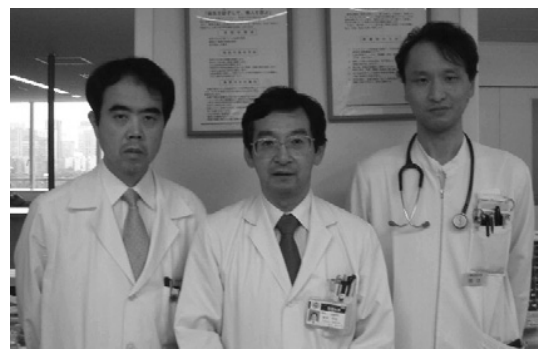

Drs Sakamoto, Hashimoto, and Taguchi (left to right). tion on microscopy.

This is frequently

found in patients with systemic lupus erythematosus (SLE). Unique echocardiographic and intracardiac findings are reported here with the technique of mitral valve plasty.

\section{Clinical Summary}

A 34-year-old woman was admitted after the sudden onset of aphasia associated with mild hemiplegia of her left arm and leg. She had been receiving treatment for SLE for 9 years, but the disease had been inactive, and she had been well, except for numbness in her left hand, at 7 months before this episode. Her medications were an $\mathrm{H}_{2}$ blocker and an oral steroid $(5 \mathrm{mg} / \mathrm{d}$ prednisolone). Admission laboratory tests revealed normal serum chemistry, normal C-reactive protein (CRP) levels, no anemia, and mild thrombocytopenia (the platelet count was $55 \times 10^{3} / \mu \mathrm{L}$ ). 